Pacific

Journal of

Mathematics

\title{
A NOTE ON FLUX INTEGRALS OVER SMOOTH REGULAR DOMAINS
}

IDO BRIGHT AND JOHN M. LEE 


\title{
A NOTE ON FLUX INTEGRALS OVER SMOOTH REGULAR DOMAINS
}

\author{
IDO BRIGHT AND JOHN M. LEE
}

\begin{abstract}
We provide new bounds on a flux integral over the portion of the boundary of one regular domain contained inside a second regular domain, based on properties of the second domain rather than the first one. This bound is amenable to numerical computation of a flux through the boundary of a domain, for example, when there is a large variation in the normal vector near a point. We present applications of this result to occupational measures and two-dimensional differential equations, including a new proof that all minimal invariant sets in the plane are trivial.
\end{abstract}

\section{Introduction}

A regular domain in $\mathbb{R}^{d}$ is a closed, embedded $d$-dimensional smooth submanifold with boundary, such as a closed ball or a closed half-space. (Throughout this paper, smooth means infinitely differentiable.) If $D \subset \mathbb{R}^{d}$ is a regular domain, its interior $\stackrel{\circ}{D}$ is an open subset of $\mathbb{R}^{d}$, and its boundary $\partial D$ is a closed, embedded, codimension-1 smooth submanifold (without boundary) which is the common topological boundary of the open sets $\stackrel{\circ}{D}$ and $\mathbb{R}^{d} \backslash D$. For this reason, the boundary of a regular domain is often called a space-separating hypersurface. The Jordan-Brouwer separation theorem (see, for example, [Guillemin and Pollack 1974, p. 89]) shows that if $S \subset \mathbb{R}^{d}$ is any compact, connected, embedded hypersurface, then the complement of $S$ has two connected components, one bounded (the interior of $S$ ) and another unbounded (the exterior of $S$ ), with $S$ as their common boundary; thus $S \cup \operatorname{Int} S$ and $S \cup$ Ext $S$ are both regular domains. But in general, the boundary of a regular domain need not be connected (for example, an annulus in the plane).

Surface integrals computing the flux through boundaries of regular domains are ubiquitous in physics and engineering. We present two bounds for surface integrals on a portion of the boundary of one domain contained inside a second domain. The results are presented for regular domains in Euclidean space for simplicity, but Theorems 1.1 and 1.2 extend to regular domains in Riemannian manifolds. See Theorem 3.3. For more details about the notation in these theorems, see Section 2.

MSC2010: primary 53A05, 58C35; secondary 28A99.

Keywords: flux integral, smooth, regular domain, occupational measure. 
Theorem 1.1. Suppose $D_{1}, D_{2} \subset \mathbb{R}^{d}$ are regular domains, such that $D_{1} \cap D_{2}$ is compact and $D_{2}$ has finite volume and surface area. Suppose $f$ is a smooth vector field defined on a neighborhood of $D_{2}$ such that $|f|$ and $|\nabla \cdot f|$ are bounded. Then the absolute value of the flux of $f$ across the portion of $\partial D_{1}$ inside $D_{2}$ satisfies

$$
\left|\int_{\partial D_{1} \cap D_{2}} f \cdot \boldsymbol{n}_{\partial D_{1}} d A\right| \leq \operatorname{Area}\left(\partial D_{2}\right)\|f\|_{\infty}+\operatorname{Vol}\left(D_{2}\right)\|\nabla \cdot f\|_{\infty} .
$$

When the vector field is divergence-free, we have the following much better bound.

Theorem 1.2. Suppose $D_{1}, D_{2} \subset \mathbb{R}^{d}$ are regular domains with compact intersection and finite surface areas, and $f$ is a smooth bounded vector field on $\mathbb{R}^{d}$ satisfying $\nabla \cdot f \equiv 0$. Then

$$
\left|\int_{\partial D_{1} \cap D_{2}} f \cdot \boldsymbol{n}_{\partial D_{1}} d A\right| \leq \frac{1}{2} \operatorname{Area}\left(\partial D_{2}\right)\|f\|_{\infty} .
$$

A surprising corollary to Theorem 1.2 bounds the integral of the normal vector of the portion of a hypersurface contained inside a second regular domain.

Corollary 1.3. Suppose $D_{1}, D_{2} \subset \mathbb{R}^{d}$ are regular domains with compact intersection and finite surface areas. The following inequality holds:

$$
\left|\int_{\partial D_{1} \cap D_{2}} \boldsymbol{n}_{\partial D_{1}} d A\right| \leq \frac{1}{2} \operatorname{Area}\left(\partial D_{2}\right) .
$$

When $D_{2}$ is convex we have the following alternative bound, which is an improvement in some cases.

Theorem 1.4. Suppose $D_{1}, D_{2} \subset \mathbb{R}^{d}$ are regular domains. If $D_{2}$ is compact and convex with diameter $\delta$, then

$$
\left|\int_{\partial D_{1} \cap D_{2}} \boldsymbol{n}_{\partial D_{1}} d A\right| \leq \frac{1}{2} \operatorname{Vol}\left(B^{d-1}(\delta / 2)\right),
$$

where $B^{d-1}(\delta / 2)$ denotes the ball in $\mathbb{R}^{d-1}$ of radius $\delta / 2$.

The significance of these results is that, although the integration is with respect to the portion of $\partial D_{1}$ inside $D_{2}$, which might have arbitrarily large surface area (see Figure 1), the bound depends only on $D_{2}$. This is due to the cancellations of the normal vector that occur in hypersurfaces that bound regular domains, and would not hold for images of general immersions of codimension 1 (see Example 4.2).

Theorem 1.1 is applicable to the numerical computation of the flux on the surface of a regular domain when there is a large variation of the normal vector near a point, resulting in a large surface area contained in a region of small volume. Indeed, the flux over the problematic part can be estimated by finding a domain containing it, avoiding direct computation. We provide an application of Corollary 1.3 in 


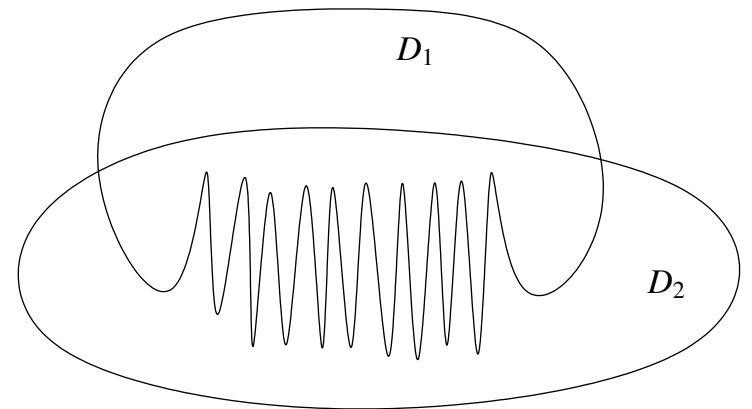

Figure 1. The setup for Theorems 1.1 and 1.2.

Section 5, for limits of sequences of regular domains with surface area increasing without bound; there we use the bound to show that in the limit, the average normal vector, say in a ball, is zero. Such a result is applied in the case $d=2$, in [Artstein and Bright 2010], to obtain a new Poincaré-Bendixson type result for planar infinite-horizon optimal control.

Corollary 1.3 generalizes a previous result, for $d=2$, established by Artstein and Bright [2010; 2013]. This topological result has proved fruitful in applications, providing new Poincaré-Bendixson type results, in an optimal-control setting [Artstein and Bright 2010; Bright 2012], and in the context of dynamics with no differentiability assumptions by Bright [2012]. The proofs of the planar result in [Artstein and Bright 2010; 2013] employ a dynamical argument, which is similar to the one used in the textbook proof of the Poincaré-Bendixson theorem. In this paper, we generalize the results to boundaries of open sets, restricting ourselves in this presentation to regular domains; however the results hold for more general sets and vector fields. The results in their fullest generality for nonsmooth domains and fluxes are presented in [Bright and Torres 2014].

Remark 1.5. The requirement that $D_{1} \cap D_{2}$ be compact is essential, as it implies that $\partial D_{1} \cap D_{2}$ is compact, so that the integrals in (1-1)-(1-3) are finite.

Remark 1.6. Theorem 1.1 can be extended, by replacing the smooth vector field $f$ with a smooth matrix-valued function $\Pi$, using the induced norm.

Remark 1.7. For simplicity, Theorem 1.2 is stated under the assumption that $f$ is defined on all of $\mathbb{R}^{d}$; but as the proof will show, if $D_{2}$ has finite volume it is only necessary that $f$ be defined on some neighborhood of $D_{2}$.

The structure of this paper is as follows. Section 2 presents notation and lemmas used in the paper. In Section 3 we prove Theorems 1.1 and 1.2, and describe how our results extend to regular domains in a Riemannian manifold. In Section 4 we prove Corollary 1.3 and Theorem 1.4, and also provide examples showing the tightness of the bound. In the last section we provide three applications of Corollary 1.3: an 
application to limits of sequences of regular domains; an extension when $d=2$; and a simplified proof of a theorem on invariant sets for dynamical systems.

\section{Notation and lemmas}

Throughout this paper, we denote the characteristic function of a set $A \subset \mathbb{R}^{d}$ by $\chi_{A}$. The $d$-dimensional volume is denoted by $\operatorname{Vol}(A)$, and the $(d-1)$-dimensional surface area of its boundary by $\operatorname{Area}(\partial A)$. Given two submanifolds $S_{1}, S_{2} \subset \mathbb{R}^{d}$, the notation $S_{1} \pitchfork S_{2}$ means that $S_{1}$ and $S_{2}$ intersect transversally. The Euclidean norm on $\mathbb{R}^{d}$ is denoted by $|\cdot|$, and the supremum norm on functions by $\|\cdot\|_{\infty}$. The divergence of a smooth vector field $f=\left(f^{1}, f^{2}, \ldots, f^{d}\right)$ at the point $x=$ $\left(x^{1}, x^{2}, \ldots, x^{d}\right) \in \mathbb{R}^{d}$ is denoted by

$$
\nabla \cdot f(x)=\frac{\partial}{\partial x^{1}} f^{1}(x)+\frac{\partial}{\partial x^{2}} f^{2}(x)+\cdots+\frac{\partial}{\partial x^{d}} f^{d}(x) .
$$

The following is a simple lemma we need for the proof of the main theorems.

Lemma 2.1. Suppose $(X, \mu)$ is a measure space, $U, V \subset X$, and $U$ has finite measure. For every real-valued function $f \in L^{\infty}(X)$, we have

$$
\begin{aligned}
& \left|\int_{U \backslash V} f(x) \mu(d x)\right| \leq \frac{1}{2}\left(\mu(U)\|f\|_{\infty}+\left|\int_{U} f(x) \mu(d x)\right|\right), \\
& \left|\int_{U \cap V} f(x) \mu(d x)\right| \leq \frac{1}{2}\left(\mu(U)\|f\|_{\infty}+\left|\int_{U} f(x) \mu(d x)\right|\right) .
\end{aligned}
$$

Proof. The first inequality follows from the triangle inequality:

$$
\begin{aligned}
\int_{U}|f(x)| \mu(d x) & \geq\left|\int_{U \backslash V} f(x) \mu(d x)\right|+\left|\int_{U \cap V} f(x) \mu(d x)\right| \\
& =\left|\int_{U \backslash V} f(x) \mu(d x)\right|+\left|\int_{U \backslash V} f(x) \mu(d x)-\int_{U} f(x) \mu(d x)\right| \\
& \geq 2\left|\int_{U \backslash V} f(x) \mu(d x)\right|-\left|\int_{U} f(x) \mu(d x)\right| .
\end{aligned}
$$

The second inequality follows by replacing $V$ with $X \backslash V$.

The proofs of the main theorems are based on the divergence theorem for certain domains in $\mathbb{R}^{d}$. Let us say a regular domain with corners in $\mathbb{R}^{d}$ is a closed subset $D \subset \mathbb{R}^{d}$ such that for each point $p \in D$, there exist an open set $U \subset \mathbb{R}^{d}$ containing $p$ and a smooth coordinate chart $\varphi: U \rightarrow \mathbb{R}^{d}$ such that $\varphi(U \cap D)$ is the intersection of $\varphi(U)$ with $\overline{\mathbb{R}}_{+}^{d}=\left\{x \in \mathbb{R}^{d} \mid x^{1} \geq 0, \ldots, x^{d} \geq 0\right\}$. Some typical examples are closed simplices and rectangular solids. Every regular domain is a regular domain with corners, and a regular domain with corners is a $d$-dimensional smooth manifold with corners in the sense defined in [Lee 2013]. 
Here is the version of the divergence theorem we will use.

Lemma 2.2. Suppose $D \subset \mathbb{R}^{d}$ is a regular domain with corners, having finite volume and surface area. If $f$ is a smooth vector field defined on $D$ such that both $|f|$ and $|\nabla \cdot f|$ are bounded, then

$$
\int_{\partial D} f \cdot \boldsymbol{n}_{\partial D} d A=\int_{D} \nabla \cdot f d V
$$

Proof. If $D$ is compact, or more generally if $f$ is compactly supported, this follows immediately from Stokes's theorem applied to the $(d-1)$-form $f\lrcorner\left(d x^{1} \wedge \cdots \wedge d x^{d}\right)$, where $\lrcorner$ denotes interior multiplication. (For Stokes's theorem on manifolds with corners, see, for example, [Lee 2013, Theorem 16.25, p. 419].) In the general case, we argue as follows. Let $\varphi:[0, \infty) \rightarrow[0,1]$ be a smooth function that is equal to 1 on $\left[0, \frac{1}{2}\right]$ and supported in $[0,1]$, and for each $r>0$ let $\varphi_{r}(x)=\varphi\left(|x|^{2} / r^{2}\right)$. Then the vector field $\varphi_{r} f$ is compactly supported, so the divergence theorem implies

$$
\int_{\partial D} \varphi_{r} f \cdot \boldsymbol{n}_{\partial D} d A=\int_{D} \nabla \cdot\left(\varphi_{r} f\right) d V .
$$

As $r \rightarrow \infty$, the integral on the left-hand side of (2-1) converges to $\int_{\partial D} f \cdot \boldsymbol{n}_{\partial D} d A$ by the dominated convergence theorem. On the other hand, for each $r>0$,

$$
\begin{aligned}
\left|\nabla \cdot\left(\varphi_{r} f\right)(x)\right| & =\left|\varphi_{r}(x) \nabla \cdot f(x)+\frac{2}{r^{2}} \sum_{i=1}^{d} \varphi^{\prime}\left(\frac{|x|^{2}}{r^{2}}\right) x^{i} f^{i}(x)\right| \\
& \leq\|\nabla \cdot f\|_{\infty}+\frac{2}{r}\left\|\varphi^{\prime}\right\|_{\infty}\|f\|_{\infty},
\end{aligned}
$$

because $|x| \leq r$ on the support of $\varphi^{\prime}\left(|x|^{2} / r^{2}\right)$. Since $\nabla \cdot\left(\varphi_{r} f\right)$ converges pointwise to $\nabla \cdot f$ and $D$ has finite volume, it follows from the dominated convergence theorem that the right-hand side of (2-1) converges to $\int_{D} \nabla \cdot f d V$.

The next proposition is used in the proof of the main theorems.

Proposition 2.3. Suppose $D_{1}$ and $D_{2}$ are regular domains in $\mathbb{R}^{d}$, with $D_{1} \cap D_{2}$ compact and with $D_{2}$ of finite volume and surface area. Suppose further that $f$ is a smooth bounded vector field defined on a neighborhood of $D_{2}$. There exists a sequence of regular domains $D_{2, i}$ such that $\partial D_{2, i}$ is transverse to $\partial D_{1}$, and the following limits hold as $i \rightarrow \infty$ :

(a) $\operatorname{Vol}\left(D_{2, i}\right) \rightarrow \operatorname{Vol}\left(D_{2}\right)$;

(b) $\operatorname{Area}\left(\partial D_{2, i}\right) \rightarrow \operatorname{Area}\left(\partial D_{2}\right)$;

(c) $\int_{\partial D_{2, i}} f \cdot \boldsymbol{n}_{\partial D_{2, i}} d A \rightarrow \int_{\partial D_{2}} f \cdot \boldsymbol{n}_{\partial D_{2}} d A$.

The domains can be chosen so that $D_{2, i}$ is either a decreasing sequence of domains whose intersection is $D_{2}$, or an increasing sequence of domains whose union is $\stackrel{\circ}{2}_{2}$. 


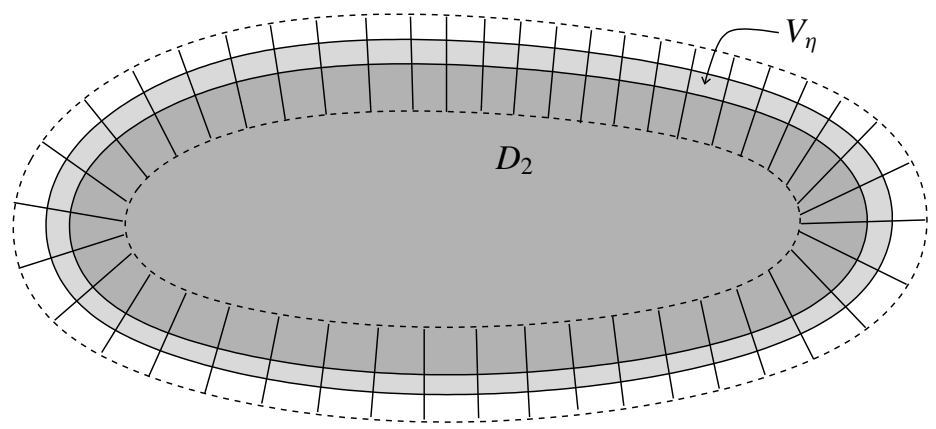

Figure 2. Defining a domain $D_{2}^{\eta}$ containing $D_{2}$.

Proof. As a smooth embedded hypersurface, $\partial D_{2}$ has a tubular neighborhood $N$, and there exists a smooth embedding $E: \partial D_{2} \times(-\delta, \delta) \rightarrow N$ such that $E(\cdot, 0)$ is the identity on $\partial D_{2}$. It can be chosen such that $E\left(\partial D_{2} \times(0, \delta)\right) \cap D_{2}=\varnothing$ and $E\left(\partial D_{2} \times(-\delta, 0]\right) \subset D_{2}$.

Let $W \subset \mathbb{R}^{d}$ be a precompact neighborhood of $D_{1} \cap D_{2}$ contained in the set on which $f$ is defined, and let $\varphi: \mathbb{R}^{d} \rightarrow[0,1]$ be a smooth compactly supported function that is equal to 1 on $\bar{W}$. For each $\eta$ such that $\delta>\eta>0$, define

$$
\begin{gathered}
V_{\eta}=\{E(x, s): 0 \leq s \leq \eta \varphi(x)\}, \\
D_{2}^{\eta}=D_{2} \cup V_{\eta} .
\end{gathered}
$$

(See Figure 2.) Then $D_{2}^{\eta}$ is a regular domain containing $D_{2}$, which agrees with $D_{2}$ outside the support of $\varphi$. Its boundary $\partial D_{2}^{\eta}$ is the image of the embedding $\iota_{\eta}$ : $\partial D_{2} \rightarrow \mathbb{R}^{d}$ given by $\iota_{\eta}(x)=E(x, \eta \varphi(x))$, which is equal to the inclusion map $\partial D_{2} \hookrightarrow \mathbb{R}^{d}$ outside supp $\varphi$. The map $E$ has full rank in $\left(\partial D_{2} \cap W\right) \times(-\delta, \delta)$, and $\varphi \equiv 1$ there, so by the parametric transversality theorem (see, for example, [Lee 2013, Theorem 6.35, p. 145]), $\partial D_{2}^{\eta}$ is transverse to $\partial D_{1}$ for almost every $\eta \in(-\delta, \delta)$.

Now let $\eta_{i}$ be a sequence of positive numbers that decreases to zero, chosen so that $\partial D_{2}^{\eta_{i}}$ is transverse to $\partial D_{1}$ for each $i$, and set $D_{2, i}=D_{2}^{\eta_{i}}$. Then $D_{2, i}$ decreases to $D_{2}$ and $\operatorname{Vol}\left(D_{2, i}\right)$ decreases to $\operatorname{Vol}\left(D_{2}\right)$. Moreover, because the embeddings $\iota_{\eta_{i}}$ converge uniformly with all derivatives to the inclusion map $\partial D_{2} \hookrightarrow \mathbb{R}^{d}$, the surface area of $\partial D_{2, i}$ converges to that of $\partial D_{2}$. Further, the function $\boldsymbol{n}_{\partial D_{2, i}} \circ \iota_{\eta_{i}}: \partial D_{2} \rightarrow \mathbb{R}^{d}$ converges to $\boldsymbol{n}_{\partial D_{2}}$. Combining these two arguments, we conclude that (c) is satisfied.

To obtain a sequence of domains that increase to $\stackrel{\circ}{D}_{2}$, we proceed instead as follows. For each $\eta$ such that $-\delta<\eta<0$, define

$$
V_{\eta}=\{E(x, s) \mid \eta \varphi(x)<s \leq 0\}, \quad D_{2}^{\eta}=D_{2} \backslash V_{\eta} .
$$

In this case, we can choose a sequence of negative numbers $\eta_{i}$ increasing to zero such that $\partial D_{2}^{\eta_{i}}$ is transverse to $\partial D_{1}$. The rest of the proof proceeds as before. 


\section{Proof of Theorems 1.1 and 1.2}

In this section, we prove Theorems 1.1 and 1.2. We start with a more general result that implies both theorems; first, we prove it when the boundaries of the domains intersect transversally, then, employing an approximation argument, we prove the general case.

Theorem 3.1. Suppose $D_{1}$ and $D_{2}$ are two regular domains in $\mathbb{R}^{d}$, such that $D_{1} \cap D_{2}$ is compact and $D_{2}$ has finite volume and surface area. Let $f$ be a smooth vector field defined on a neighborhood of $D_{2}$ such that both $|f|$ and $|\nabla \cdot f|$ are bounded. The absolute value of the flux of $f$ across the portion of $\partial D_{1}$ inside $D_{2}$ satisfies the following bound:

$$
\left|\int_{\partial D_{1} \cap D_{2}} f \cdot \boldsymbol{n}_{\partial D_{1}} d A\right|
$$

$$
\leq \frac{1}{2}\left(\operatorname{Area}\left(\partial D_{2}\right)\|f\|_{\infty}+\left|\int_{\partial D_{2}} f \cdot \boldsymbol{n}_{\partial D_{2}} d A\right|+\operatorname{Vol}\left(D_{2}\right)\|\nabla \cdot f\|_{\infty}+\left|\int_{D_{2}} \nabla \cdot f d V\right|\right) .
$$

The same estimate holds when $\partial D_{1} \cap D_{2}$ is replaced by $\partial D_{1} \cap \stackrel{\circ}{D}_{2}$ on the lefthand side.

Proposition 3.2. Theorem 3.1 holds when $\partial D_{1} \pitchfork \partial D_{2}$.

Proof. Note that $\partial\left(D_{1} \cap D_{2}\right)$ is compact, and

$$
\partial\left(D_{1} \cap D_{2}\right)=\left(\partial D_{1} \cap D_{2}\right) \cup\left(D_{1} \cap \partial D_{2}\right) .
$$

Adding and subtracting $\int_{\partial D_{2} \cap D_{1}} f \cdot \boldsymbol{n}_{\partial D_{2}} d A$, we obtain

$$
\begin{aligned}
\int_{\partial D_{1} \cap D_{2}} f \cdot \boldsymbol{n}_{\partial D_{1}} d A & =\int_{\partial D_{1} \cap D_{2}} f \cdot \boldsymbol{n}_{\partial D_{1}} d A+\int_{\partial D_{2} \cap D_{1}} f \cdot \boldsymbol{n}_{\partial D_{2}} d A-\int_{\partial D_{2} \cap D_{1}} f \cdot \boldsymbol{n}_{\partial D_{2}} d A \\
& =\int_{\partial\left(D_{1} \cap D_{2}\right)} f \cdot \boldsymbol{n}_{\partial\left(D_{1} \cap D_{2}\right)} d A-\int_{\partial D_{2} \cap D_{1}} f \cdot \boldsymbol{n}_{\partial D_{2}} d A,
\end{aligned}
$$

since $\partial D_{1} \cap \partial D_{2}$ is a smooth $(d-2)$-dimensional submanifold and thus has zero $(d-1)$-dimensional area.

The assumption $\partial D_{1} \pitchfork \partial D_{2}$ implies that $D_{1} \cap D_{2}$ is a smooth manifold with corners. To see this, we just need to show that each point is contained in the domain of an appropriate smooth coordinate chart. For points not in $\partial D_{1} \cap \partial D_{2}$, this follows easily from the fact that $D_{1}$ and $D_{2}$ are regular domains. If $x \in \partial D_{1} \cap \partial D_{2}$, we can find a local defining function $u^{1}$ for $D_{1}$ such that $D_{1}$ is locally given by the equation $u^{1} \geq 0$; and similarly we can find a local defining function $u^{2}$ for $D_{2}$. The assumption $\partial D_{1} \pitchfork \partial D_{2}$ ensures that $d u^{1}$ and $d u^{2}$ are linearly independent at $x$. Thus we can find smooth functions $u^{3}, \ldots, u^{d}$ such that $\left(u^{1}, \ldots, u^{d}\right)$ form the required local coordinates in a neighborhood of $x$. 
Applying the divergence theorem, we get

$$
\int_{\partial D_{1} \cap D_{2}} f \cdot \boldsymbol{n}_{\partial D_{1}} d A=\int_{D_{2} \cap D_{1}} \nabla \cdot f d V-\int_{\partial D_{2} \cap D_{1}} f \cdot \boldsymbol{n}_{\partial D_{2}} d A .
$$

Applying Lemma 2.1 to both terms on the right hand side completes the proof for $\partial D_{1} \cap D_{2}$. The result for $\partial D_{1} \cap \stackrel{\circ}{D}_{2}$ is immediate in this case, because $\partial D_{1} \cap \partial D_{2}$ has zero surface area.

Proof of Theorem 3.1. Let $D_{2, i}$ be a sequence of regular domains decreasing to $D_{2}$ and satisfying the conclusions of Proposition 2.3. By Proposition 3.2, for every $i$ we have that $\left|\int_{\partial D_{1} \cap D_{2, i}} f \cdot \boldsymbol{n}_{\partial D_{1}} d A\right|$ is bounded by

$\frac{1}{2}\left(\operatorname{Area}\left(\partial D_{2, i}\right)\|f\|_{\infty}+\left|\int_{\partial D_{2, i}} f \cdot \boldsymbol{n}_{\partial D_{2, i}} d A\right|+\operatorname{Vol}\left(D_{2, i}\right)\|\nabla \cdot f\|_{\infty}+\left|\int_{D_{2, i}} \nabla \cdot f d V\right|\right)$.

Proposition 2.3 shows that the first three terms above converge to the first three terms on the right-hand side of (3-1). To complete the proof, we use the facts that the sets $D_{2, i}$ decrease to $D_{2}$ and the compact sets $\partial D_{1} \cap D_{2, i}$ decrease to $\partial D_{1} \cap D_{2}$ as $i$ goes to infinity, and thus the Lebesgue dominated convergence theorem yields

$$
\lim _{i \rightarrow \infty}\left|\int_{D_{2, i}} \nabla \cdot f d V\right|=\left|\int_{D_{2}} \nabla \cdot f d V\right|
$$

and

$$
\lim _{i \rightarrow \infty}\left|\int_{\partial D_{1} \cap D_{2, i}} f \cdot \boldsymbol{n}_{\partial D_{1}} d A\right|=\left|\int_{\partial D_{1} \cap D_{2}} f \cdot \boldsymbol{n}_{\partial D_{1}} d A\right| .
$$

This completes the proof for $\partial D_{1} \cap D_{2}$.

To prove the estimate for $\partial D_{1} \cap \stackrel{\circ}{D}_{2}$, we use the same argument, but with $D_{2, i}$ chosen to increase to $\stackrel{\circ}{D}_{2}$. Because $\partial D_{2}$ has $d$-dimensional measure zero, we have $\int_{\dot{D}_{2}} \nabla \cdot f d V=\int_{D_{2}} \nabla \cdot f d V$, and the result follows.

Proof of Theorem 1.1. Inequality (1-1) follows immediately from (3-1) and obvious estimates for the integrals.

Proof of Theorem 1.2. We first assume that $\operatorname{Vol}\left(D_{2}\right)<\infty$, so that (3-1) holds. In this case, the last two terms in (3-1) are zero because $\nabla \cdot f=0$, and the second term is zero by the divergence theorem.

Now consider the case in which $D_{2}$ has infinite volume. Let $D_{2}^{\prime}$ denote the closure of $\mathbb{R}^{d} \backslash D_{2}$, which is a regular domain with interior $\stackrel{\circ}{2}_{2}^{\prime}=\mathbb{R}^{d} \backslash D_{2}$. Because $\operatorname{Area}\left(\partial D_{2}^{\prime}\right)=\operatorname{Area}\left(\partial D_{2}\right)<\infty$, the isoperimetric inequality (see [De Giorgi 1953]) implies that $D_{2}^{\prime}$ has finite volume. If $D_{1}$ also has finite volume, the divergence theorem gives

$$
\int_{\partial D_{1} \cap D_{2}} f \cdot \boldsymbol{n}_{\partial D_{1}} d A+\int_{\partial D_{1} \cap{ }_{D_{2}^{\prime}}^{\prime}} f \cdot \boldsymbol{n}_{\partial D_{1}} d A=\int_{\partial D_{1}} f \cdot \boldsymbol{n}_{\partial D_{1}} d A=\int_{D_{1}} \nabla \cdot f d V=0,
$$


and (1-2) follows from Theorem 3.1 applied to the second term on the left-hand side above. On the other hand, if $\operatorname{Vol}\left(D_{1}\right)=\infty$, we let $D_{1}^{\prime}$ be the closure of $\mathbb{R}^{d} \backslash D_{1}$ (which has finite volume), and apply the above argument with $D_{1}^{\prime}$ in place of $D_{1}$.

To conclude this section, we explain what modifications need to be made to Theorems 1.1 and 1.2 and their proofs to adapt them to the case of regular domains in Riemannian manifolds.

Suppose $M$ is a $d$-dimensional smooth Riemannian manifold with Riemannian metric $g$ and volume density $d V_{g}$. (If $M$ is oriented, $d V_{g}$ can be interpreted as a differential $d$-form; but otherwise it needs to be interpreted as a density. See [Lee 2013, pp. 427-434] for basic properties of densities.) A regular domain $D \subset M$ is defined just as in the case $M=\mathbb{R}^{d}$. If $D \subset M$ is a regular domain, it has a uniquely defined outward unit normal vector field $\boldsymbol{n}_{\partial D}$. For any such domain, we let $\tilde{g}$ denote the induced Riemannian metric on $\partial D$, and let $d A_{\tilde{g}}$ denote its volume density.

For any smooth vector field $f$ defined on an open subset of $M$, the divergence of $f$, denoted by $\nabla \cdot f$, is defined as follows. If $M$ is oriented, then $\nabla \cdot f$ is the unique vector field that satisfies $\left.(\nabla \cdot f) d V_{g}=d(f\lrcorner d V_{g}\right)$. On a nonorientable manifold, we define it locally by choosing an orientation and using the same formula; because $\nabla \cdot f$ is unchanged when the orientation is reversed, it is globally defined. The divergence theorem then holds in exactly the same form for smooth $d$-dimensional submanifolds with corners in $M$. Moreover, any compact smooth embedded hypersurface in $M$ has a tubular neighborhood in $M$. (See [Bredon 1993, Theorem 11.14, p. 100] for a proof. Although the proof there is for manifolds embedded in Euclidean space, it follows from the Whitney embedding theorem that it applies to all smooth manifolds.)

Using these facts, the proof of the following theorem is carried out exactly like the proofs of Theorems 1.1 and 1.2. To avoid complications, we restrict to the case in which $D_{2}$ is compact.

Theorem 3.3. If $D_{1}$ and $D_{2}$ are regular domains in a Riemannian manifold $(M, g)$ with $D_{2}$ compact, and $f$ is a smooth vector field defined on a neighborhood of $D_{2}$, then the conclusions of Theorems 1.1 and 1.2 hold, namely,

$$
\left|\int_{\partial D_{1} \cap D_{2}}\left\langle f, \boldsymbol{n}_{\partial D_{1}}\right\rangle_{g} d A_{\tilde{g}}\right| \leq \operatorname{Area}\left(\partial D_{2}\right)\|f\|_{\infty}+\operatorname{Vol}\left(D_{2}\right)\|\nabla \cdot f\|_{\infty},
$$

and if $\nabla \cdot f \equiv 0$,

$$
\left|\int_{\partial D_{1} \cap D_{2}}\left\langle f, \boldsymbol{n}_{\partial D_{1}}\right\rangle_{g} d A_{\tilde{g}}\right| \leq \frac{1}{2} \operatorname{Area}\left(\partial D_{2}\right)\|f\|_{\infty}
$$

\section{Bounding integrals of normal fields}

In this section, we prove Corollary 1.3 and Theorem 1.4. We also provide examples on the tightness of the bound. 
Proof of Corollary 1.3. Let $v=\int_{\partial D_{1} \cap D_{2}} \boldsymbol{n}_{\partial D_{1}} d A$. If $|v|=0$ there is nothing to prove, so we assume that $|v|>0$, and let $f: \mathbb{R}^{d} \rightarrow \mathbb{R}^{d}$ be the constant vector field $f \equiv v /|v|$. Clearly, $|v|=v \cdot v /|v|=\int_{\partial D_{1} \cap D_{2}} f \cdot \boldsymbol{n}_{\partial D_{1}} d A$. Now, since $\nabla \cdot f \equiv 0$ and $\|f\|_{\infty}=1$, the proof follows from Theorem 1.2.

To prove Theorem 1.4, we begin with a lemma.

Lemma 4.1. Suppose $D \subset \mathbb{R}^{d}$ is a compact convex regular domain with diameter $\delta$ and $C$ is any measurable subset of $\partial D$. Then for any unit vector $v \in \mathbb{R}^{d}$, we have

$$
\int_{C} v \cdot \boldsymbol{n}_{\partial D} d A \leq \frac{1}{2} \operatorname{Vol}\left(B^{d-1}(\delta / 2)\right)
$$

Proof. First consider the case $v=e_{d}=(0, \ldots, 0,1)$. After applying a translation, we can assume that $D$ is contained in the set where $x^{d} \geq 0$. Its boundary is the union of the three subsets $\partial D_{+}, \partial D_{0}$, and $\partial D_{-}$, defined as the subsets of $\partial D$ where $v \cdot \boldsymbol{n}_{\partial D}$ is positive, zero, or negative, respectively.

Now, let $A$ be the following subset of $\mathbb{R}^{d}$ :

$$
A=\left\{\left(x^{1}, \ldots, x^{d-1}, t x^{d}\right) \mid\left(x^{1}, \ldots, x^{d}\right) \in D, 0 \leq t \leq 1\right\} .
$$

Then $A$ is a compact convex set, and its boundary is the union of the three subsets $\partial A_{+}, \partial A_{0}$, and $\partial A_{-}$, defined in the same way as above. (See Figure 3.)

The fact that $D$ is convex ensures that $\partial A_{+}=\partial D_{+}, \partial A_{0} \supset \partial D_{0}$, and $\partial A_{-}$is contained in the hyperplane where $x^{d}=0$. Moreover, $A$ is a $C^{1}$ manifold with corners. (Its boundary might not be smooth at points where $\partial A_{0}$ meets $\overline{\partial A}_{+}$, but it is at least $C^{1}$ there.)

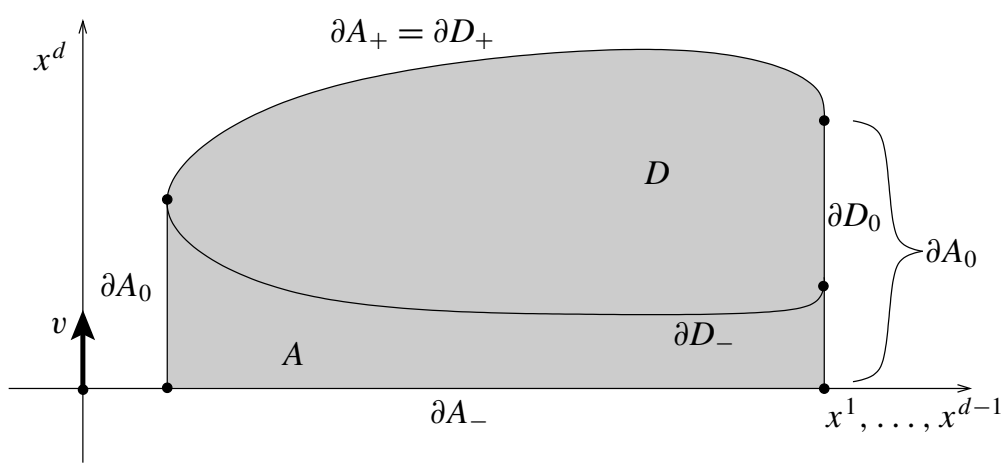

Figure 3. Proof of Lemma 4.1. 
Using the fact that $v \cdot \boldsymbol{n}_{\partial D}<0$ on $\partial D_{-}$and $v \cdot \boldsymbol{n}_{\partial D}=0$ on $\partial D_{0}$, we compute

$$
\begin{aligned}
\int_{C} v \cdot \boldsymbol{n}_{\partial D} d A & =\int_{C \cap \partial D_{+}} v \cdot \boldsymbol{n}_{\partial D} d A+\int_{C \cap \partial D_{0}} v \cdot \boldsymbol{n}_{\partial D} d A+\int_{C \cap \partial D_{-}} v \cdot \boldsymbol{n}_{\partial D} d A \\
& \leq \int_{C \cap \partial D_{+}} v \cdot \boldsymbol{n}_{\partial D} d A \leq \int_{\partial D_{+}} v \cdot \boldsymbol{n}_{\partial D} d A \\
& =\int_{\partial A_{+}} v \cdot \boldsymbol{n}_{\partial A} d A=-\int_{\partial A_{-}} v \cdot \boldsymbol{n}_{\partial A} d A,
\end{aligned}
$$

where in the last line we have used the divergence theorem for the vector field $f \equiv v$ and the fact that $v \cdot \boldsymbol{n}_{\partial A}=0$ on $\partial A_{0}$. Since $\boldsymbol{n}_{\partial A}=-v$ on $\partial A_{-}$, the last integral is equal to the area of $\partial A_{-}$. Since $\partial A_{-}$is contained in a $(d-1)$-dimensional ball of radius $\delta / 2$, the result follows.

Finally, for the case of a general unit vector $v$, we just apply a rotation to $D$ and apply the above argument.

Proof of Theorem 1.4. Let $D_{1}$ and $D_{2}$ be as in the statement of the theorem. If $\int_{\partial D_{1} \cap D_{2}} \boldsymbol{n}_{\partial D_{1}} d A=0$, there is nothing to prove, so assume the integral is nonzero, and let $v$ be the unit vector in the direction of $\int_{\partial D_{1} \cap D_{2}} \boldsymbol{n}_{\partial D_{1}} d A$. Then

$$
\begin{aligned}
\left|\int_{\partial D_{1} \cap D_{2}} \boldsymbol{n}_{\partial D_{1}} d A\right| & =v \cdot \int_{\partial D_{1} \cap D_{2}} \boldsymbol{n}_{\partial D_{1}} d A \\
& =\int_{\partial D_{1} \cap D_{2}} v \cdot \boldsymbol{n}_{\partial D_{1}} d A,
\end{aligned}
$$

and the result follows from Lemma 4.1.

The following examples demonstrate the tightness of the bound for nonconvex sets, as well as the necessity of the condition that the hypersurface be the boundary of a regular domain.

Example 4.2. The main theorem explicitly uses the divergence theorem, which is applied to space-separating hypersurfaces. In fact, the bounds do not apply for images of general smooth immersions. To construct a counterexample in the plane (i.e., for $d=2$ ), start with a smooth Jordan curve in the plane, then cover it $m$ times, with small perturbations, making the integral on the left-hand side of (1-3) roughly $m$ times as large, while the right-hand side is fixed because it depends only on $\partial D_{2}$. Clearly, whenever the left-hand side of (1-3) is not zero, we can choose $m$ large enough that the inequality does not hold.

Example 4.3. To see that the bound obtained in Corollary 1.3 is tight, and cannot be replaced by a bound based only on the diameter of $D_{2}$ when $D_{2}$ is not convex, we consider comb-shaped subsets of $\mathbb{R}^{d}$, for $d \geq 2$, generated in the following manner. Fix $n>2$, and let $D_{n}$ be a closed nonsmooth comb-shaped set defined as 


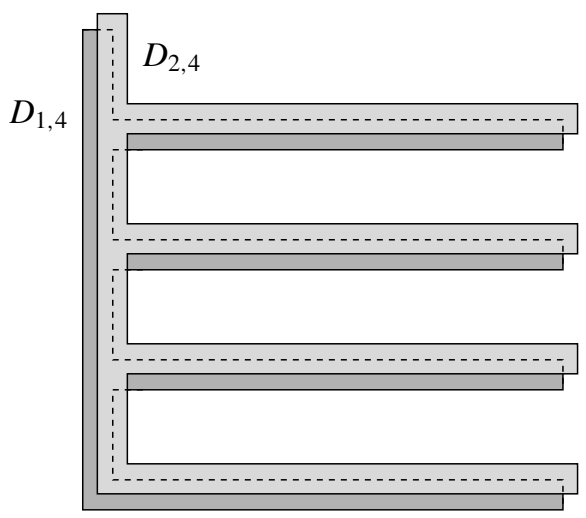

Figure 4. The domains of Example 4.3 in the case $n=4$ (before smoothing).

the union of the following rectangles:

$$
\begin{aligned}
R_{i, n} & =\left\{\left(x^{1}, \ldots, x^{d}\right) \in[0,1]^{d} \mid i / n \leq x^{2} \leq i / n+1 / n^{2}\right\}, \quad 0 \leq i \leq n-1 ; \\
R_{n, n} & =\left\{\left(x^{1}, \ldots, x^{d}\right) \in[0,1]^{d} \mid 0 \leq x^{1} \leq 1 / n^{2}\right\} .
\end{aligned}
$$

Applying a small perturbation we then smooth its corners, and set $D_{1, n}$ accordingly. Let $D_{2, n}$ be the translation of $D_{1, n}$ by the vector $\left(1 /\left(2 n^{2}\right), 1 /\left(2 n^{2}\right), 0, \ldots, 0\right) \in \mathbb{R}^{d}$. (See Figure 4.) By our construction, the surface area of each set $\partial D_{1, n}$ or $\partial D_{2, n}$ is roughly $2 n+2$, and the fraction of the boundary area where the normal vector of $\partial D_{1, n}$ is parallel to the $x^{2}$-axis is roughly $n /(n+1)$, approaching 1 when $n$ is large. Notice that by the choice of $D_{2, n}$, when we integrate the normal vector in the portion of $\partial D_{1, n}$ inside $D_{2, n}$ we capture only the part pointing in the positive direction of the $x^{2}$-axis. This shows that the integral of the normal vector has magnitude of roughly $n$, approaching half the surface area when we take $n$ to infinity.

\section{Applications: limits of hypersurfaces \& planar results}

In this section we provide two applications of Corollary 1.3, extending previous planar results in [Artstein and Bright 2010; 2013]. The first is for limits of regular domains whose surface areas increase without bound. The second is an application in the planar case.

Corollary 1.3 bounds the normal vector of the boundary of a regular domain in a second regular domain, by the surface area of the boundary of the second domain, and completely disregarding the surface area of the original hypersurface. This is now applied to surfaces with increasing surface area, establishing a new result on the limit.

We will denote by $\mathbb{S}^{d-1} \subset \mathbb{R}^{d}$ the unit $(d-1)$-sphere. For every hypersurface we define a corresponding probability measure using the following notation: 
Definition 5.1. Suppose $S \subset \mathbb{R}^{d}$ is a smooth hypersurface endowed with a unit normal vector field $\boldsymbol{n}_{S}$. We define the empirical measure $\mu \in P\left(\mathbb{R}^{d} \times \mathbb{S}^{d-1}\right)$ corresponding to $S$ by

$$
\mu(U \times V)=\frac{1}{\operatorname{Area}(S)} \int_{S \cap U} \chi_{V}\left(\boldsymbol{n}_{S}\right) d A,
$$

for all open sets $U \subset \mathbb{R}^{d}$ and $V \subset \mathbb{S}^{d-1}$.

A useful property of empirical measures is that, if $f: \mathbb{R}^{d} \times \mathbb{S}^{d-1} \rightarrow \mathbb{R}$ is continuous, we have

$$
\frac{1}{\operatorname{Area}(S)} \int_{S} f\left(x, \boldsymbol{n}_{S}\right) d A=\int_{\mathbb{R}^{d} \times \mathbb{S}^{d-1}} f(x, n) \mu(d x, d n) .
$$

We endow the set of probability measures $P\left(\mathbb{R}^{d} \times \mathbb{S}^{d-1}\right)$ with the weak topology, namely, a sequence of measures $\mu_{1}, \mu_{2}, \ldots \in P\left(\mathbb{R}^{d} \times \mathbb{S}^{d-1}\right)$ converges to a measure $\mu_{0} \in P\left(\mathbb{R}^{d} \times \mathbb{S}^{d-1}\right)$ if for every bounded continuous function $g(x, n)$,

$$
\int_{\mathbb{R}^{d} \times \mathbb{S}^{d-1}} g(x, n) \mu_{0}(d x, d n)=\lim _{i \rightarrow \infty} \int_{\mathbb{R}^{d} \times \mathbb{S}^{d-1}} g(x, n) \mu_{i}(d x, d n) .
$$

Another tool we need for the next theorem is disintegration of measures. Given a probability measure $\mu \in P\left(\mathbb{R}^{d} \times \mathbb{S}^{d-1}\right)$, we define its marginal measure, $p(d x)$, as the projection on $\mathbb{R}^{d}$, namely, $p(A)=\mu\left(A \times \mathbb{S}^{d-1}\right)$ for every measurable set $A \subset \mathbb{R}^{d}$. Also, we denote the measure valued function $\mu^{x}(d n)$, the disintegration of $\mu$ with respect to $p$, for $p$-almost every $x$. With this notation, for every pair of measurable sets $U \subset \mathbb{R}^{d}$ and $V \subset \mathbb{S}^{d-1}$, we have that

$$
\mu(U \times V)=\int_{U} \mu^{x}(V) p(d x) .
$$

We now state the main result regarding the limits of regular domains.

Theorem 5.2. Let $D_{1}, D_{2}, \ldots \subset \mathbb{R}^{d}$ be a sequence of compact regular domains, such that the surface areas of their boundaries increases to infinity. If the empirical measures $\mu_{1}, \mu_{2}, \ldots$, corresponding to the sequence $\partial D_{1}, \partial D_{2}, \ldots$, converge weakly to $\mu_{0}$, then

$$
h(x)=\int_{\mathbb{S}^{d-1}} n \mu_{0}^{x}(x)(d n)=0
$$

for $p_{0}$-almost every $x$, where $\mu_{0}(d x, d n)=p_{0}(d x) \mu_{0}^{x}(d n)$ is the disintegration of $\mu_{0}$ with respect to its projection $p_{0}$.

Proof. Let $B=B(x, r) \subset \mathbb{R}^{d}$ be a ball centered at $x$ with radius $r>0$. By the definition of the empirical measures and by Corollary 1.3,

$$
\left|\int_{B \times \mathbb{S}^{d-1}} n d \mu_{i}(d x, d n)\right|=\left|\frac{1}{\operatorname{Area}\left(\partial D_{i}\right)} \int_{\partial D_{i} \cap B} \boldsymbol{n}_{\partial D_{i}} d A\right| \leq \frac{\operatorname{Area}(\partial B)}{2 \operatorname{Area}\left(\partial D_{i}\right)} .
$$


Weak convergence of measures and the dominated convergence theorem imply that

$$
\begin{aligned}
\left|\int_{B \times \mathbb{S}^{d-1}} n d \mu_{0}(d x, d n)\right| & =\lim _{i \rightarrow \infty}\left|\int_{B \times \mathbb{S}^{d-1}} n d \mu_{i}(d x, d n)\right| \\
& \leq \lim _{i \rightarrow \infty} \frac{\operatorname{Area}(\partial B)}{2 \operatorname{Area}\left(\partial D_{i}\right)}=0
\end{aligned}
$$

for a set of values of $r>0$ of full measure for which $\mu_{i}\left(\partial B(x, r) \times \mathbb{S}^{d-1}\right)=0$, for all $i=0,1,2, \ldots$ Using the disintegration notation we obtain that

$$
\begin{aligned}
\left|\int_{B \times \mathbb{S}^{d-1}} n d \mu_{0}(d x, d n)\right| & =\left|\int_{B}\left(\int_{\mathbb{S}^{d-1}} n \mu_{0}^{x}(d n)\right) p_{0}(d x)\right| \\
& =\int_{B} h(x) p_{0}(d x)=0
\end{aligned}
$$

for almost every ball $B$. If the measure $p_{0}(d x)$ is Lebesgue measure, by the Lebesgue differentiation theorem we have $h(x)=0$ almost everywhere. The LebesgueBesicovitch differentiation theorem extends this result to Radon measures (see, for example, [Evans and Gariepy 1992, p. 43]).

Remark 5.3. Theorem 5.2 requires the convergence of the empirical measures. When the domains in the sequence are contained in some compact set $K$, the compactness of the space $K \times \mathbb{S}^{d-1}$ implies the compactness of $P\left(K \times \mathbb{S}^{d-1}\right)$, and, therefore, the existence of a converging subsequence [Billingsley 1999, p. 72].

In two dimensions, our result extends as follows.

Corollary 5.4. Suppose $x_{1}:\left[0, \tau_{1}\right] \rightarrow \mathbb{R}^{2}$ is a parametrized smooth Jordan curve and $D_{2} \subset \mathbb{R}^{2}$ is a regular domain. If the length of $\partial D_{2}$ is $L_{2}$, then

$$
\left|\int_{0}^{\tau_{1}} \chi_{D_{2}}\left(x_{1}(t)\right) \frac{d}{d t} x_{1}(t) d t\right| \leq \frac{L_{2}}{2} .
$$

Proof. Let $T_{1}$ and $N_{1}$ be the unit tangent and normal vectors of $x_{1}$. Using the arc-length parametrization, we have that

$$
\left|\int_{0}^{\tau_{1}} \chi_{D_{2}}\left(x_{1}(t)\right) \frac{d}{d t} x_{1}(t) d t\right|=\left|\int_{0}^{L_{1}} \chi_{D_{2}}\left(x_{1}(s)\right) T_{1}(s) d s\right|,
$$

where $L_{1}$ is the length of $x_{1}$. Expressing the tangent vector in terms of the normal vector, we reduce the previous expression to

$$
\left|\int_{0}^{L_{1}} \chi_{D_{2}}\left(x_{1}(s)\right)\left[\begin{array}{rr}
0 & -1 \\
1 & 0
\end{array}\right] N_{1}(s) d s\right|=\left|\int_{0}^{L_{1}} \chi_{D_{2}}\left(x_{1}(s)\right) N_{1}(s) d s\right|,
$$

as the rotation matrix is orthogonal. Applying Corollary 1.3 completes the proof. 
For our final application, we consider an ordinary differential equation in the plane defined by

$$
\frac{d x}{d t}=f(x)
$$

where $f: \mathbb{R}^{2} \rightarrow \mathbb{R}^{2}$ is a vector field (generally assumed at least Lipschitz continuous). An invariant set for $f$ is a subset of $\mathbb{R}^{2}$ that is invariant under the forward flow of $f$ and a minimal set is a nonempty closed invariant set that is minimal with respect to inclusions. A trivial minimal set is a set that is the image of either a stationary solution or a periodic solution.

We present a new short proof of the following well-known result.

Theorem 5.5. Suppose $f$ is a smooth vector field on $\mathbb{R}^{2}$. Then every minimal set for $f$ is trivial.

The textbook proof of this theorem (see [Verhulst 1996]) relies on the PoincaréBendixson theorem, and employs dynamical arguments. Here we present a simpler proof based on the divergence theorem, and specifically on Corollary 1.3. Note that the divergence theorem was used by Bendixson in the proof of the Bendixson criterion, which verifies that no periodic solutions exist.

Our proof uses the following well-known lemmas.

Lemma 5.6. Suppose $\Omega \subset \mathbb{R}^{2}$ is a minimal set for (5-1) and $x^{*}:[0, \infty) \rightarrow \mathbb{R}^{2}$ is a solution to (5-1) with trajectory contained in $\Omega$. For every $y_{0} \in \Omega, s \in[0, \infty)$, and $\delta>0$, there exists $t>s$ such that $\left|x^{*}(t)-y_{0}\right|<\delta$.

Proof. Suppose the lemma does not hold for some $y_{0}, s$, and $\delta$. Then the curve $y^{*}(t)=x^{*}(s+t)$ is a solution to (5-1) with trajectory contained in $\Omega \backslash B\left(y_{0}, \delta\right)$ for a suitable $\delta>0$, in contradiction to the minimality of $\Omega$.

The next lemma follows easily from Sard's theorem.

Lemma 5.7. Suppose $I \subset \mathbb{R}$ is a compact interval and $g: I \rightarrow \mathbb{R}$ is smooth. Then for almost every $r \in \mathbb{R}$, the set $g^{-1}(r)=\{t \in I \mid g(t)=r\}$ is finite.

Proof of Theorem 5.5. Clearly, $\Omega$ is a singleton if and only if it contains a point $y \in \Omega$ such that $f(y)=0$, so we may assume henceforth that $f$ does not vanish in $\Omega$ and $\Omega$ contains more than one point. Choose $D>0$ such that $\Omega \backslash B\left(x^{*}(0), 3 D\right) \neq \varnothing$. We construct sequences of real numbers $\left\{\delta_{i}\right\}$ and $\left\{t_{i}\right\}$, and a sequence of simple closed curves $\left\{\gamma_{i}\right\}$, as follows. Set $\delta_{0}=D$, and let $t_{0}$ be the first time where $x^{*}$ meets $\partial B\left(x^{*}(0), \delta_{0}\right)$. For $i=1,2, \ldots$ do the following:

(a) Choose $\delta_{i}<\delta_{i-1} / 2$ small enough that $\left|x^{*}(0)-x^{*}(t)\right|>\delta_{i}$ for all $t \in\left[t_{0}, t_{i-1}\right]$.

(b) Let $t_{i}$ be the first time after $t_{0}$ where the curve $x^{*}$ meets $\partial B\left(x^{*}(0), \delta_{i}\right)$. (Here we use Lemma 5.6.) 


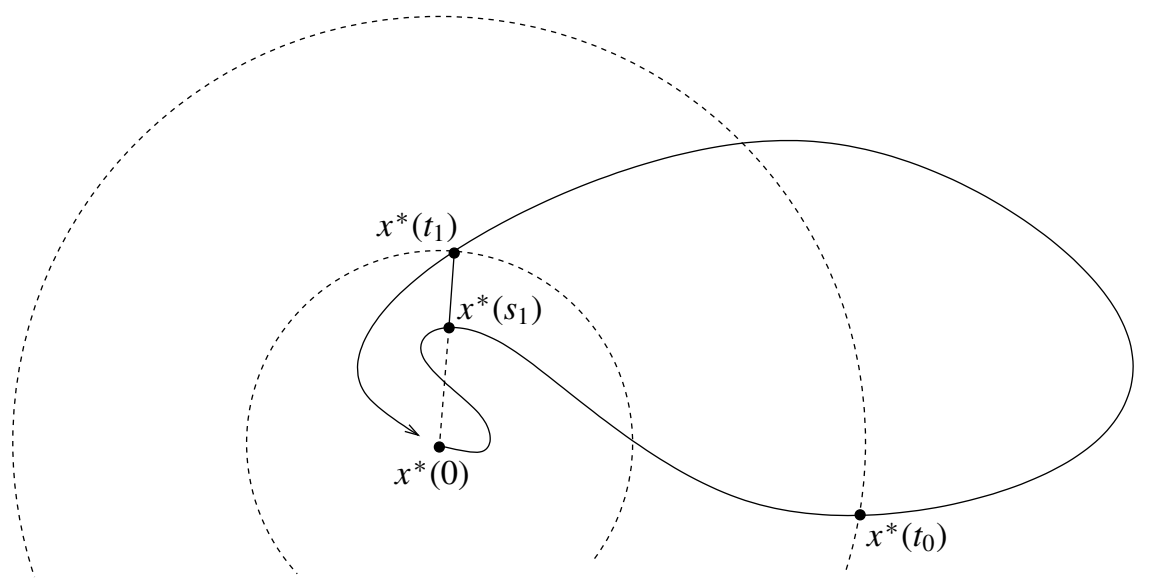

Figure 5. Proof of Theorem 5.5.

(c) Starting from $x^{*}\left(t_{i}\right)$, follow the line connecting it to $x^{*}(0)$, until that line first meets a point in $x^{*}\left(\left[0, t_{0}\right]\right)$. Let $x^{*}\left(s_{i}\right)$ be this point. (See Figure 5.)

(d) Let $\gamma_{i}$ be the parametrized piecewise smooth curve obtained by following the curve $x^{*}$ in the interval $\left[s_{i}, t_{i}\right]$, and then the line connecting its endpoints with unit speed.

Note that $t_{i}$ is an increasing sequence and that the uniqueness of the solution with respect to the initial condition implies that every $\gamma_{i}$ is a Jordan curve. Suppose first that the sequence $\left\{t_{i}\right\}$ is bounded above. Then $t_{i} \rightarrow t^{*} \in \mathbb{R}^{+}$and $x^{*}\left(t_{i}\right) \rightarrow x^{*}\left(t^{*}\right)$. According to our construction, $\left|x^{*}(0)-x^{*}\left(t_{i}\right)\right|=\delta_{i}<2^{-i} D$ for every $i$. Hence by continuity $x^{*}\left(t^{*}\right)=x^{*}(0)$, and $x^{*}$ is periodic. By the minimality of $\Omega$, the image of $x^{*}$ is $\Omega$.

The only remaining possibility is $t_{i} \nearrow \infty$. Fix $y_{0} \in \Omega$ such that $\left|y_{0}-x^{*}(0)\right|>2 D$. By Lemma 5.7, there exists arbitrarily small $r_{0}<D$ such that the set

$$
\left\{t \in[0, s]|| x^{*}(t)-y_{0} \mid=r_{0}\right\}
$$

is finite for every $s>0$. (This follows from the fact that $g(t)=\left|x^{*}(t)-y_{0}\right|^{2}$ is a smooth function of $t$.) Note that this implies that the portion of $\gamma_{i}$ in $B_{0}=B\left(y_{0}, r_{0}\right)$ is part of the trajectory $x^{*}$, and that for every $i$ the Jordan curve $\gamma_{i}$ intersects $\partial B_{0}$ at a finite number of points.

For every $i$, we let $D_{i}$ denote the domain consisting of the Jordan curve $\gamma_{i}$ together with its interior. Although $D_{i}$ is not a regular domain, it is a regular domain with two corner points, which are outside of $\bar{B}_{0}$, and it is easy to see that Corollary 1.3 can be applied to $\partial D_{i} \cap B_{0}$. Thus by Corollary 5.4,

$$
\left|\int_{\left\{t \leq t_{i} \mid x^{*}(t) \in B_{0}\right\}} \frac{d}{d t} x^{*}(t) d t\right|=\left|\int_{\left\{t \leq t_{i} \mid x^{*}(t) \in B_{0}\right\}} f\left(x^{*}(t)\right) d t\right| \leq \pi r_{0} .
$$


Because $\Omega$ is minimal, Lemma 5.6 implies that the set $\left\{t \mid x^{*}(t) \in B_{0}\right\}$ has infinite measure. This implies that 0 is contained in the convex hull of the set

$$
\left\{f(y) \mid y \in \bar{B}_{0}\right\} .
$$

The radius $r_{0}$ can be chosen arbitrary small; therefore, the continuity of $f$ implies that $f\left(y_{0}\right)=0$, in contradiction.

\section{Acknowledgements}

The first author wishes to thank Vered Rom-Kedar for suggesting the idea of studying the integral of the normal vector, and Monica Torres for valuable remarks.

This work was supported by the Applied Mathematics Program within the Department of Energy (DOE) Office of Advanced Scientific Computing Research.

\section{References}

[Artstein and Bright 2010] Z. Artstein and I. Bright, "Periodic optimization suffices for infinite horizon planar optimal control”, SIAM J. Control Optim. 48:8 (2010), 4963-4986. MR 2011k:49008 Zbl 1208.49006

[Artstein and Bright 2013] Z. Artstein and I. Bright, "On the velocity of planar trajectories", NoDEA Nonlinear Differential Equations Appl. 20:2 (2013), 177-185. MR 3046971 Zbl 06196734

[Billingsley 1999] P. Billingsley, Convergence of probability measures, 2nd ed., Wiley, New York, 1999. MR 2000e:60008 Zbl 0944.60003

[Bredon 1993] G. E. Bredon, Topology and geometry, Graduate Texts in Mathematics 139, Springer, New York, 1993. MR 94d:55001 Zbl 0791.55001

[Bright 2012] I. Bright, "A reduction of topological infinite-horizon optimization to periodic optimization in a class of compact 2-manifolds", J. Math. Anal. Appl. 394:1 (2012), 84-101. MR 2926206 Zbl 1244.49004

[Bright and Torres 2014] I. Bright and M. Torres, "The integral of the normal and fluxes over sets of finite perimeter", 2014. To appear in Interfaces Free Bound.

[De Giorgi 1953] E. De Giorgi, "Definizione ed espressione analitica del perimetro di un insieme", Atti Accad. Naz. Lincei. Rend. Cl. Sci. Fis. Mat. Nat. (8) 14 (1953), 390-393. MR 15,20b Zbl 0051.29403

[Evans and Gariepy 1992] L. C. Evans and R. F. Gariepy, Measure theory and fine properties of functions, CRC, Boca Raton, FL, 1992. MR 93f:28001 Zbl 0804.28001

[Guillemin and Pollack 1974] V. Guillemin and A. Pollack, Differential topology, Prentice-Hall, Englewood Cliffs, NJ, 1974. Reprinted by AMS Chelsea, Providence, RI, 2010. MR 50 \#1276 Zbl 0361.57001

[Lee 2013] J. M. Lee, Introduction to smooth manifolds, 2nd ed., Graduate Texts in Mathematics 218 , Springer, New York, 2013. MR 2954043 Zbl 1258.53002

[Verhulst 1996] F. Verhulst, Nonlinear differential equations and dynamical systems, Springer, Berlin, 1996. MR 97g:34003 Zbl 0854.34002

Received October 13, 2013. Revised November 26, 2013. 
IDO BRIGHT

DEPARTMENT OF APPLIED MATHEMATICS

UNIVERSITY OF WASHINGTON

SEATTLE, WA 98195-3925

UNITED STATES

ibright@uw.edu

JOHN M. LEE

DEPARTMENT OF MATHEMATICS

UNIVERSITY OF WASHINGTON

SEATTLE, WA 98195-4350

UNITED STATES

johnmlee@uw.edu 


\title{
PACIFIC JOURNAL OF MATHEMATICS
}

\author{
msp.org/pjm
}

Founded in 1951 by E. F. Beckenbach (1906-1982) and F. Wolf (1904-1989)

\section{EDITORS}

Don Blasius (Managing Editor)

Department of Mathematics

University of California

Los Angeles, CA 90095-1555

blasius@math.ucla.edu

\author{
Paul Balmer \\ Department of Mathematics \\ University of California \\ Los Angeles, CA 90095-1555 \\ balmer@math.ucla.edu \\ Robert Finn \\ Department of Mathematics \\ Stanford University \\ Stanford, CA 94305-2125 \\ finn@math.stanford.edu \\ Sorin Popa \\ Department of Mathematics \\ University of California \\ Los Angeles, CA 90095-1555 \\ popa@math.ucla.edu
}

\author{
Vyjayanthi Chari \\ Department of Mathematics \\ University of California \\ Riverside, CA 92521-0135 \\ chari@math.ucr.edu \\ Kefeng Liu \\ Department of Mathematics \\ University of California \\ Los Angeles, CA 90095-1555 \\ liu@math.ucla.edu \\ Jie Qing \\ Department of Mathematics \\ University of California \\ Santa Cruz, CA 95064 \\ qing@ cats.ucsc.edu
}

\section{PRODUCTION}

Silvio Levy, Scientific Editor, production@msp.org

\section{SUPPORTING INSTITUTIONS}

ACADEMIA SINICA, TAIPEI

CALIFORNIA INST. OF TECHNOLOGY

INST. DE MATEMÁTICA PURA E APLICADA

KEIO UNIVERSITY

MATH. SCIENCES RESEARCH INSTITUTE

NEW MEXICO STATE UNIV.

OREGON STATE UNIV.

\author{
STANFORD UNIVERSITY \\ UNIV. OF BRITISH COLUMBIA \\ UNIV. OF CALIFORNIA, BERKELEY \\ UNIV. OF CALIFORNIA, DAVIS \\ UNIV. OF CALIFORNIA, LOS ANGELES \\ UNIV. OF CALIFORNIA, RIVERSIDE \\ UNIV. OF CALIFORNIA, SAN DIEGO \\ UNIV. OF CALIF., SANTA BARBARA
}

\author{
Daryl Cooper \\ Department of Mathematics \\ University of California \\ Santa Barbara, CA 93106-3080 \\ cooper@math.ucsb.edu \\ Jiang-Hua Lu \\ Department of Mathematics \\ The University of Hong Kong \\ Pokfulam Rd., Hong Kong \\ jhlu@maths.hku.hk \\ Paul Yang \\ Department of Mathematics \\ Princeton University \\ Princeton NJ 08544-1000 \\ yang@math.princeton.edu
}

These supporting institutions contribute to the cost of publication of this Journal, but they are not owners or publishers and have no responsibility for its contents or policies.

See inside back cover or msp.org/pjm for submission instructions.

The subscription price for 2014 is US $\$ 410 /$ year for the electronic version, and \$535/year for print and electronic.

Subscriptions, requests for back issues and changes of subscribers address should be sent to Pacific Journal of Mathematics, P.O. Box 4163, Berkeley, CA 94704-0163, U.S.A. The Pacific Journal of Mathematics is indexed by Mathematical Reviews, Zentralblatt MATH, PASCAL CNRS Index, Referativnyi Zhurnal, Current Mathematical Publications and Web of Knowledge (Science Citation Index).

The Pacific Journal of Mathematics (ISSN 0030-8730) at the University of California, c/o Department of Mathematics, 798 Evans Hall \#3840, Berkeley, CA 94720-3840, is published twelve times a year. Periodical rate postage paid at Berkeley, CA 94704, and additional mailing offices. POSTMASTER: send address changes to Pacific Journal of Mathematics, P.O. Box 4163, Berkeley, CA 94704-0163.

PJM peer review and production are managed by EditFLOW ${ }^{\circledR}$ from Mathematical Sciences Publishers.

\section{PUBLISHED BY}

\section{mathematical sciences publishers \\ nonprofit scientific publishing}

http://msp.org/

(C) 2014 Mathematical Sciences Publishers 


\section{PACIFIC JOURNAL OF MATHEMATICS}

Volume $272 \quad$ No. $2 \quad$ December 2014

Marginally trapped submanifolds in space forms with arbitrary

signature

HENRI ANCIAUX

One line complex Kleinian groups

275

Waldemar Barrera, Angel Cano and JuAn Pablo

NAVARRETE

A note on flux integrals over smooth regular domains

305

IDO BRIGHT and JOHN M. LEE

On stable commutator length in hyperelliptic mapping class groups

323

DANny Calegari, NaOyuKi Monden and Masatoshi Sato

Compositions of birational endomorphisms of the affine plane

PierRetTe CASSOU-NoguÈs and DANIEl DAigle

The Bochner formula for isometric immersions

395

ALESSANDRO SAVO

On solutions to Cournot-Nash equilibria equations on the sphere

423

MICAH WARREN

Double and triple Givental's J-functions for stable quotients invariants

439

ALEKSEY ZiNGER

Acknowledgement 